4. - - Paracompact spaces 29, Bull. Amer Math. Soc. Research Problem 61-6-29.

5. J. Nagata, On a necessary and sufficient condition of metrizability, J. Inst. Polytech., Osaka City Univ., Ser. A. Math. vol. 1 (1950) pp. 93-100.

6. Yu. Smirnov, A necessary and sufficient condition for metrizability of a topological space, Doklady Akad. Nauk. SSSR. N.S. vol. 77 (1951) pp. 197-200.

7. G. T. Whyburn, Open and closed mappings, Duke Math. J. vol. 17 (1950) pp. 69-74.

\title{
UNIVERSITY OF WASHINGTON
}

\section{COMPACT MAPPINGS}

EDWIN HALFAR

In the Memoir Open mappings on locally compact spaces, G. T. Whyburn shows the equivalence of his definition of compact mappings and that of E. A. Vainstein [1] under the assumption that the spaces involved are separable and metric. In the following, it is shown that the two are equivalent on more general spaces provided that the range space is suitably restricted. The remainder of the note is concerned with a result about the relation between closedness of a mapping and continuity and with an elaboration of a result concerning quasi-interior mappings.

In general, the spaces are $T_{1}$ unless otherwise stated. The mapping (i.e. continuous function) $f(X)=Y$ is closed if $F$ a closed subset of $X$ implies $f(F)$ is closed; $f$ is compact if $K$ a compact subset of $Y$ implies $f^{-1}(K)$ is a compact subset of $X ; f$ is quasi-interior if for any $y \in Y$ and any open set $U$ in $X$ which contains a compact component of $f^{-1}(y), y$ is interior to $f(U) . A$ is an inverse set if $A=f^{-1}(A)$. Also, $G_{0}$ will denote the union of all sets of the type $f^{-1}(y)$ which are subsets of $G$.

Theorem 1. If $f(X)=Y$ is a closed mapping and if for each $y \in Y$, $f^{-1}(y)$ is compact, then $f$ is compact.

Let $K$ be a compact subset of $Y$ and $g$ a covering of $f^{-1}(K)$. Since $f$ is closed, for each open subset $G$ of $X, G_{0}$ is an open inverse set, and $f\left(G_{0}\right)$ is an open subset of $Y$. For some $y \in K, f^{-1}(y)$ may fail to be a subset of a single element of $\mathcal{G}$, but since $f^{-1}(y)$ is compact, a finite subcollection $\mathcal{G}_{1}$ of $g$ will cover $f^{-1}(y)$. Let $G^{*}$ be the union of the elements of $g_{1}$. Then $G_{0}^{*}$ is a non-null open inverse set. Thus, the collec-

Received by the editors July 9, 1956 and, in revised form, December 5, 1956. 
tion of sets of the form $f\left(G_{0}\right)$ and $f\left(G_{0}^{*}\right)$ respectively constitutes a covering of $K$ which is reducible to a finite subcovering. Hence a finite number of the sets $G_{0}$ and $G_{0}^{*}$ cover $f^{-1}(K)$ so that since each $G_{0}^{*}$ is a subset of a finite union of elements of $\mathcal{G}$, there is finite subcover from S of $f^{-1}(K)$.

Theorem 2. If $f(X)=Y$ is a compact mapping and $Y$ is a locally compact Hausdorff space, then $f$ is closed and point inverses are compact.

That point inverses are compact is obvious.

Let $F$ be a closed subset of $X$, and suppose $y \notin f(F)$ and is an accumulation point of $f(F)$. Since $Y$ is locally compact, an open set $G$ exists such that $y \in G$ and $\bar{G}$ is compact. Now $f(F) \cap \bar{G}$ cannot be compact, for if it is, there exist open neighborhoods $U$ and $V$ of $f(F) \cap \bar{G}$ and $y$ respectively with $U \cap V=\varnothing$. Then $G \cap V \cap f(F) \subset V \cap \bar{G} \cap f(F)$ $=\varnothing$, a contradiction since $y \in \mathrm{Cl}[f(F)]$.

Since $\bar{G}$ is compact, $f^{-1}(\bar{G})$ is compact and $F \cap f^{-1}(\bar{G})$ is a non-null compact subset of $X$. From this it follows that $f\left[F \cap f^{-1}(\bar{G})\right]=f(F) \cap \bar{G}$ is compact. This a contradiction and $y \in f(F)$.

Corollary. A necessary and sufficient condition that a mapping $f(X)=Y$ where $Y$ is a locally compact Hausdorff space be compact is that $f$ be closed and that for each $y \in Y, f^{-1}(y)$ be a compact subset of $X$.

In these results, the continuity of $f$ has formed a part of the hypotheses. Under certain conditions, the other restrictions on $f$ may imply that $f$ is continuous.

THeOREM 3. If $X$ is a regular space and $Y$ is compact, then if $f(X)$ $=Y$ is closed with closed point inverses, $f$ is continuous.

Suppose $f$ is not continuous at $x \in X$. There exists, then, a neighborhood $V$ of $f(x)=y$ such that for every neighborhood $U$ of $x$, $f(U) \cap(Y-V) \neq \varnothing$. Since $Y$ is compact, $Y-V$ and $f(\bar{U})$ are closed, it follows that $f(\bar{U}) \cap(Y-V)$ is compact. Also, the collection $\{f(\bar{U}) \cap(Y-V)\}$ has the finite intersection property, for if a finite number of $U$ 's existed such that $\bigcap_{j=1}^{j=n}\left\{f\left(\bar{U}_{j}\right) \cap(Y-V)\right\}=\varnothing$, then $(Y-V) \cap f\left(\bigcap_{j=1}^{j=n} U_{j}\right) \subset(Y-V) \cap f\left[\mathrm{Cl}\left(\bigcap_{j=1}^{j=n} U_{j}\right)\right] \subset \bigcap_{j=1}^{j=n}\left\{f\left(\bar{U}_{j}\right) \cap(Y-V)\right\}$ $=\varnothing$ so that $f$ would be continuous. Hence the intersection of all the sets of the form $f(\bar{U}) \cap(Y-V)$ is non-null, and there is a $y^{\prime}$ in this intersection with $y^{\prime} \neq y$. Then $x \notin f^{-1}\left(y^{\prime}\right)$, and since $f^{-1}\left(y^{\prime}\right)$ is closed, open sets $U_{1}$ and $U_{2}$ exist such that $U_{1} \cap U_{2}=\varnothing ; x \in U_{1}$ and $f^{-1}\left(y^{\prime}\right)$ $\subset U_{2}$. It follows that $y^{\prime} \notin f\left(\bar{U}_{1}\right)$, but since $x \in U_{1}, f\left(\bar{U}_{1}\right) \cap(Y-V) \neq \varnothing$ and $y^{\prime} \in f\left(\bar{U}_{1}\right)$, a contradiction. Therefore $f$ is continuous.

In the discussion of the properties of quasi-interior mappings in 
[2], it was shown that, in general, local compactness and local connectedness are not invariant under such mappings. However, the following theorem exhibits a modification under which these invariant properties are regained.

THEOREM 4. If $X$ is locally connected (locally compact), and $f$ is a quasi-interior mapping such that for each $y \in f(X)=Y$, Hausdorff, $f^{-1}(y)$ contains a compact component, then $Y$ is locally connected (locally compact).

Let $y \in Y$; $V$ be any open set with $y \in V$ and $R$ be the component of $V$ containing $y$. If $z \in R$, then $f^{-1}(z)$ contains a compact component $C$. If $X$ is locally connected, there is a component $G$ of $f^{-1}(V)$ such that $G$ is open and $C \subset G$. Hence $z$ is interior to $f(G) \subset V$, and since $f(G)$ is connected and $R \cap f(G) \neq \varnothing, f(G) \subset R$ so that $z$ is interior to $R$.

Let $D$ be a compact component of $f^{-1}(y)$. If $X$ is locally compact, there is an open set $U$ such that $\bar{U}$ is compact and $D \subset U$. It follows then that $y$ is in the interior of $f(U)$ and an open set $G$ exists such that $y \in G \subset \bar{G} \subset \mathrm{Cl}[f(U)]=f(\bar{U})$. Thus $Y$ is locally compact.

\section{REFERENCES}

1. E. A. Vainstein, On closed mappings of metric spaces, C. R. (Doklady) Acad. Sci. USSR. N. S. vol. 57 (1947) p. 319.

2. G. T. Whyburn, Open mappings on locally compact spaces, Memoirs of the American Mathematical Society, no. 1, 1950.

UNIVERSITY OF NEBRASKA 